\title{
Feasibility of a physical activity pathway for Irish primary care physiotherapy services
}

\author{
Emer M. Barrett ${ }^{\mathrm{a}, *}$, Juliette Hussey ${ }^{\mathrm{a}}$, Catherine D. Darker ${ }^{\mathrm{b}}$ \\ a Discipline of Physiotherapy, School of Medicine, Trinity College Dublin, St James's Hospital, Dublin 8, Ireland \\ ${ }^{\mathrm{b}}$ Department of Public Health and Primary Care, Trinity College Dublin, Tallaght Hospital, Dublin 24, Ireland
}

\begin{abstract}
Objectives To establish consensus on a physical activity pathway suitable for use by physiotherapists in Irish primary care. The physical activity pathway "Let's Get Moving" was examined to agree recruitment criteria and seek consensus on component parts.

Design Modified Delphi approach which attempts to achieve a convergence of opinion, over a series of iterations. Three rounds of questionnaires were used.

Setting Primary care.

Participants 41 senior physiotherapists working in primary care for a median of 6 years (IQR 3.7 to 8.5).

Main outcome measures Statements achieving consensus; defined as at least $70 \%$ of participants scoring a 6 or a 7 , indicating high agreement, on a 7 point Likert scale.

Results The response rate was $98 \%$. There was a high degree of consensus for many components of the pathway. Participants agreed that all patients attending physiotherapy should be eligible for recruitment onto the pathway as well as accepting referrals from other health professionals and direct access from the public. Private physiotherapists highlighted concerns about recruiting fee paying patients onto the pathway. The pathway should be integrated into other preventative and chronic disease programmes in primary care. Modifications to the original pathway included the use of a pedometer in addition to the General Practice Physical Activity Questionnaire. Training needs in physical activity screening and motivational interviewing, as well as additional staffing were identified to support implementation.

Conclusions The Physical Activity Pathway "Let's Get Moving" was accepted as a clinically feasible resource to primary care physiotherapists with some modifications and with the support of additional resources.
\end{abstract}

(C) 2016 Chartered Society of Physiotherapy. Published by Elsevier Ltd. All rights reserved.

Keywords: Physical activity; Primary care; Physical activity pathway; Physical activity promotion; Physical activity screening

\section{Introduction}

The World Health Organisation ranks physical inactivity as the fourth leading cause of death globally and a leading risk factor for the development of non-communicable diseases (NCDs) [1]. In 2010 it was estimated that $87 \%$ of all deaths in Ireland were due to NCDs [1] and the number of Irish adults with a chronic disease is expected to rise by $40 \%$ between 2007 and 2020 [2]. Results from the national health survey

\footnotetext{
* Correspondence: Tel.: +3531 896 2120; fax: +3531 4531915 .

E-mail addresses: barrete@tcd.ie (E.M. Barrett),jmhussey@tcd.ie (J. Hussey), darkerc@tcd.ie (C.D. Darker).
}

have found that only one-third of the Irish population are considered sufficiently active to meet physical activity (PA) recommendations [3]. In response to these challenges the Governmental Health Strategy has set a national target to increase by $20 \%$ the proportion of people undertaking regular PA by 2025 [4].

For over a decade health services in Ireland have been undergoing significant reform. This has seen the expansion of primary care services with increased focus on health promotion with the aim of reducing the onset of chronic disease [4]. As a result of these reforms, increasing numbers of physiotherapists are being employed in publically funded primary care services which offer additional opportunities to target PA 


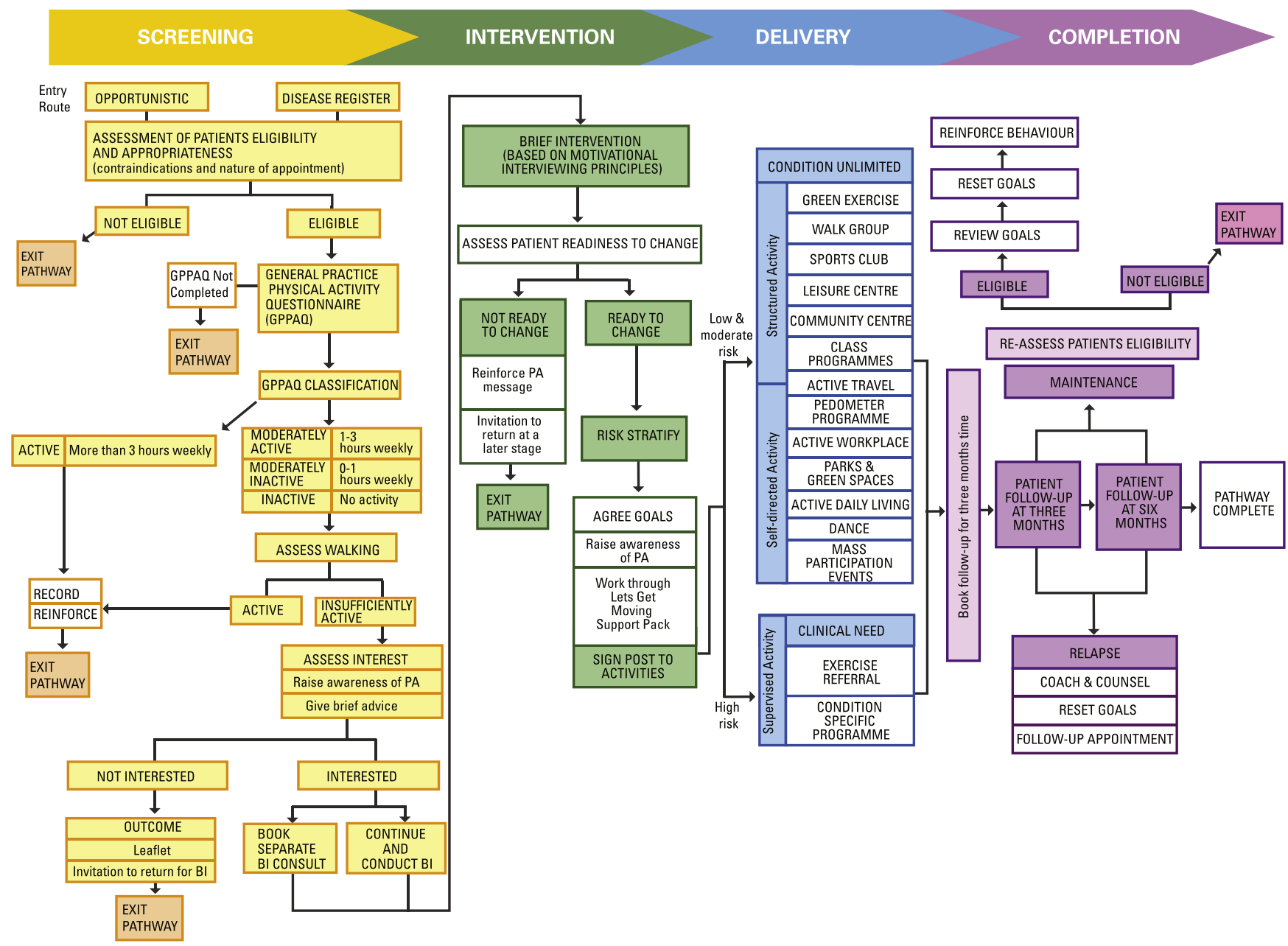

Fig. 1. Let's Get Moving Physical Activity Pathway.

in primary care. Public physiotherapy services are generally accessed directly by patients and in addition to this private physiotherapy services are available to fee paying patients.

Previous work has highlighted the need to establish a systematic and evidence based approach to screening and promoting PA within Irish primary care physiotherapy services [5]. Such an approach would seek to combine formalised screening methods, together with a number of recommended PA interventions and be applicable to the wide spectrum of patients seen in primary care. One method of doing this is through the use of a PA pathway.

The PA pathway "Let's Get Moving" (Fig. 1) was developed by the Department of Health in England in collaboration with National Health Service London (NHS) and Natural England [6]. It is based on the National Institute for Health and Care Excellence (NICE) Public Health Guidance "Four Commonly Used Methods to Promote Physical Activity" [7]. This guidance, which was recently updated, endorses the use of brief PA interventions in primary care as being clinically effective and economically efficient in the long term $[7,8]$.
The PA care pathway consists of five key stages; recruitment, screening, intervention, delivery and completion. It is a means of systematically recruiting and screening patients PA levels to determine if they are meeting the public health guidelines [9]. Individuals not meeting the guidelines are offered a brief intervention based on the principles of motivational interviewing and supported by other recommended strategies such as written materials and follow up appointments $[8,10,11]$. Based on their risk and personal preferences, individuals are then signposted to local PA opportunities which can include structured activities such as exercise classes, self directed activities such as pedometer programmes and condition specific classes such as weight management.

A process evaluation of the pathway carried out in six general practices in London found the pathway to be feasible for delivery in primary care and specific recommendations for its implementation were made [12]. Similar feasibility studies have investigated its use in the West Midlands in the UK and in Scotland [13,14]. Whilst concluding that introduction of the pathway was feasible certain issues such as variability in 
implementation, the availability of resources and workforce development needs were identified as potentially impacting on its effectiveness.

Whilst the pathway was designed so that it can flexibly be adapted to fit within existing services [6] it has yet to be established whether this model is suitable for use in health systems outside of the UK and by professions such as physiotherapists who were not included in the feasibility studies [12-14].

This research investigated the clinical feasibility of introducing this pathway for use by primary care physiotherapists in Ireland. Physiotherapists were asked to review the components of the pathway in the context of their own role, expertise and services to determine, whether the pathway as a model, was the most appropriate fit for their service. The overall aim of this study was to agree, a PA pathway suitable for use by physiotherapists in Irish primary care services.

The specific objectives were to:

1. Agree criteria for recruitment onto the PA pathway;

2. Establish consensus for each of the component parts of the pathway and;

3. Determine if there were additional training needs and resources required to support its implementation.

\section{Methodology}

This study utilised a modified Delphi approach. This process attempts to achieve a convergence of opinion among experts on a specific topic, over a series of rounds or iterations $[15,16]$. A three round Delphi questionnaire was proposed for this study.

\section{Panel}

Purposive sampling was used to identify a panel of 40 expert physiotherapists. Inclusion criteria included primary care physiotherapists, working in the province of Leinster in the Republic of Ireland, employed in a senior position with a minimum of three years experience. Representation from clinical specialists, physiotherapy managers and senior physiotherapists working in public health services and private practice were sought. Physiotherapists who did not fit the inclusion criteria or were unable to commit to the Delphi process were excluded.

\section{Study procedure}

During the month of April 2013, the manager of five physiotherapy departments meeting the inclusion criteria were contacted by the researcher to request participation. Interested staff attended an introductory education session delivered by the researcher at participants' place of work. The education session provided details about the PA pathway and the Delphi process.

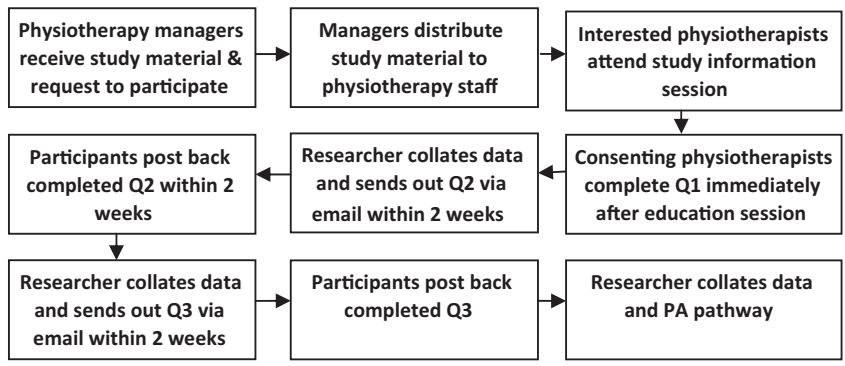

Fig. 2. Flowchart of data collection process.

A flow chart representing the data collection process is presented in Fig. 2. Participants received one email reminder to return the questionnaire during each round. Stamped addressed envelopes were provided. Data collection occurred between May and June 2013 and took a total of eight weeks.

\section{Questionnaires and Delphi methodology}

The first round questionnaire was semi structured and consisted of seven sections. Section one questioned participants on the best method of recruiting patients onto the PA pathway. Sections two to five were designed to address each of the four component parts of the pathway; screening, intervention, delivery and completion. Section six examined the feasibility of introducing the pathway to clinical practice and requested training needs and additional resources required to support its delivery. The final section of the questionnaire collated demographic and professional details.

In each section of the questionnaire participants were asked to indicate their agreement/disagreement with the inclusion of each step of the PA pathway using a seven point Likert scale; where one indicated a low level of agreement (do not agree) and seven indicated a high level of agreement (highly agree). Open ended questions at the end of each section enabled participants to make additional comments or suggest alternative options if appropriate.

Rounds two and three took the form of structured questionnaires incorporating feedback and new statements generated from the open ended questions of the preceding round. Feedback consisted of the overall anonymised rankings of the group for each statement, together with a copy of the participant's initial responses. The second and third round questionnaires followed the same format as the round one questionnaire with participants asked to indicate their agreement/disagreement with each statement using the Likert scale. Statements which received consensus in the preceding round were removed from subsequent rounds.

\section{Data management and analysis}

Data from each round of questionnaires were assigned a numerical code, inputted and analysed using SPSS version 19.0 (SPSS, Inc., Evanston, IL). Each round of data was cleaned twice to identify any errors or omissions. 
Descriptive statistics were used to examine each statement for consensus. The level of consensus was determined by calculating the median, interquartile range (IQR) and the percentage agreement with each statement. Consensus was defined as at least $70 \%$ of participants scoring either a six or a seven, on a seven point Likert scale, where the median was equal to six or seven, and the IQR was less than or equal to one.

The use of the median and IQR rather than the mean and standard deviation are recommended for use in the Delphi methodology [15]. The IQR is a measure of statistical dispersion, and is the difference between the third and first quartiles. A level of consensus where the IQR $\leq 1$ represents an agreement level where $50 \%$ of the observations fall within one point on a measure.

Content analysis techniques, verified by a second researcher, were used to analyse the qualitative data received from the open ended questions of round one and used to generate new statements for inclusion in round two. Kappa statistics were used to determine consistency between raters and ranged from $0.891(p<0.001)$ to $0.966(p<0.001)$ for each question indicating the highest level of agreement [17].

\section{Results}

Overall, 43 physiotherapists who attended the introductory sessions consented to participate. Two physiotherapists did not meet the inclusion criteria leaving a final sample size of 41. The demographic and professional details of participants are presented in Table 1.

Results from the three round Delphi process are presented in Table S1. Results are based on responses from 41 participants in rounds one and two and 40 participants in round three. Overall 28 of 43 (65\%) statements achieved consensus

Table 1

Demographic and professional details.

\begin{tabular}{ll}
\hline Panel professional details & $\mathbf{N}=\mathbf{4 1}$ \\
\hline Gender $n(\%)$ & $2(5)$ \\
\hline Male & $39(95)$ \\
Female & $11(27)$ \\
\hline Years since qualification $n(\%)$ & $17(42)$ \\
\hline 5 to 10 & $8(19)$ \\
11 to 15 & $5(12)$ \\
16 to 20 & \\
$>20$ & $34(83)$ \\
\hline Employment grade $n$ (\%) & $4(10)$ \\
\hline Senior & $3(7)$ \\
Manager & \\
Clinical Specialist & $33(80)$ \\
\hline Public or private employment $n$ (\%) & $8(20)$ \\
\hline Public & $9(22)$ \\
Private Practice & $32(78)$ \\
\hline MSc that included exercise prescription $n$ (\%) & $6(3.7$ to 8.5$)$ \\
\hline Yes & \\
\hline No &
\end{tabular}

in round one and thus were incorporated into the final pathway. No section of the pathway achieved full consensus on this round.

Supplementary Table S1 related to this article can be found, in the online version, at http://dx.doi.org/10.1016/ j.physio.2016.02.001.

The qualitative analysis of open ended questions from round one generated 20 new statements which were incorporated into round two and three questionnaires. These are presented as the shaded areas in Table 1. Nine of these statements related to additional training and resources required to deliver the programme. The remainder suggested amendments or additions to the recruitment, screening and completion sections of the pathway. There were no additional statements regarding the intervention or delivery components of the pathway. An additional minor theme generated, concerned private physiotherapists who identified that it may be difficult to deliver this pathway to a patient who was paying to attend physiotherapy for treatment of a specific condition or injury. It was also reported that arranging follow up assessments might be problematic due to the financial nature of their appointments and that offering the pathway as a separate financial package may be an option to overcome this.

Overall 19 of $34(56 \%)$ statements achieved consensus in round two including all of those in the delivery section of the pathway. Eleven of the $14(78 \%)$ remaining statements achieved consensus on the final round including all of statements concerned with screening and feasibility. All but three items failed to reach consensus. A summary of the main findings, addressed under the three study objectives is presented in Table 2 and will be discussed in the following section.

\section{Discussion}

Results from this study would suggest that overall the Let's Get Moving PA pathway was accepted as a clinically feasible resource by Irish primary care physiotherapists. Participants established consensus on recruitment criteria and suggested a number of modifications or additions to the original pathway, particularly to the screening and completion stages. Additional staffing, training and patient resources were identified as necessary to support its implementation.

\section{Recruitment criteria}

Participants agreed that all patients attending physiotherapy should be eligible for recruitment onto the pathway as well as accepting referrals from other health professionals and direct access from the public. Active promotion of the pathway and its integration into other preventative or chronic disease programmes in primary care, such as weight management and diabetes was encouraged. This would suggest that primary care physiotherapists valued an active and targeted approach to recruitment rather than the opportunistic recruitment of patients already attending physiotherapy 
Table 2

Summary of main study findings.

\section{Agree recruitment criteria} RECRUITMENT

Pathway should be open to all patients attending physiotherapy Referrals from other health professionals and directly from the public appropriate Pathway should be incorporated into other preventative or chronic disease programmes

May be difficult to recruit fee paying patients

2. Establish consensus on component parts of pathway SCREENING

\section{GPPAQ accepted as a screening tool} Use of pedometers and additional health risk questionnaires appropriate Brief interventions may be carried out as part of routine physiotherapy appointments or booked as separate appointments

INTERVENTION Consensus reached on all components of original pathway

DELIVERY Consensus reached on all components of original pathway

COMPLETION Consensus reached on all components of original pathway

May be appropriate to follow up some patients more frequently than 3 and 6 months

Coaching of patients who relapse should be dependent on their motivation to change

3. Identify additional training needs and resources to support implementation CLINICAL

Pathway useful and feasible to practice FEASIBILITY interviewing and population screening required

Additional physiotherapy and administrative staff required to support pathway

Additional patient resources required to support pathway

services. Previous work in England found that the opportunistic approach yielded very low recruitment rates which were estimated as $6 \%$ of all potential primary care consultations [12]. Recruiting patients via disease registers was more successful, although response rates varied ranging from $9 \%$ to $59 \%$ [12]. Disease registers were not identified as a potential mode of entry by participants in this study. This may reflect the limited availability of such registers outside of the acute sector in Ireland, as well as the lack of shared electronic health records in primary care, limiting the access of physiotherapists to patient records for targeted recruitment [18].

Participants did however identify the opportunity to integrate the PA pathway into planned and existing chronic disease programmes in primary care. Chronic disease management programmes were initially identified as a priority by the Irish government in 2008 as part of its overall strategy

to re-orientate the health services towards a more integrated model of care [19]. At present in Ireland, this model of care is at its most advanced in diabetes management. The current provision for PA under this programme includes the assessment of PA under "review lifestyle factors" but does not include guidance on the use of a formal assessment. Whilst specific PA recommendations for patients with diabetes are included, there is no clear guidance on who is responsible for this aspect of a patient's care or on how to manage high risk patients in need of supervised exercise referral. The inclusion of the PA pathway as part of an overall strategy to manage diabetes and other chronic diseases could systematically guide clinicians through the process of integrating PA into these coordinated packages of care. Similar approaches have been described in Scotland where the pathway is incorporated into long term condition reviews as well as lifestyle and health promotion courses including smoking cessation and weight management [14].

The final major finding in this section relates to the concerns expressed by private physiotherapists about recruiting fee paying patients onto the pathway. Attendance at private physiotherapy clinics is paid for either by the patient personally, or for those with private medical insurance, approximately half the cost of each appointment may be reimbursed for a set number of visits each year. At present, the majority of people still attend physiotherapy in response to an injury or for treatment of a medical condition, so it will require a significant mind shift on the part of both the public and the practitioner to establish health promotion as an equally relevant activity. This will necessitate action on several fronts including public advertising, the provision of competitively priced packages of care targeting PA promotion and actively advocating for insurers to include health promotion as part of their range of benefits.

\section{Consensus on pathway components}

There were a number of modifications or additions suggested to the original pathway including the use of a pedometer and an additional health risk questionnaire for use with the General Practice Physical Activity Questionnaire (GPPAQ). The GPPAQ was accepted as a suitable assessment by physiotherapists supporting previous research which found it acceptable for use in general practice, with GPs and practice nurses finding it easy to use and quick to administer [20,21]. Infrequent use of formalised screening tools has previously been described amongst physiotherapists in Ireland [5] and adoption of the GPPAQ as part of the overall PA pathway would offer the opportunity to improve on this. The suggestion of the use of a pedometer in addition to the questionnaire may reflect the working patterns of physiotherapists who generally provide more prolonged treatment over a number of visits, with increased opportunity to incorporate more detailed screening methods into their practice.

Amongst the completion components of the pathway, one of the suggested alterations was that some patients may 
benefit from more frequent follow up than the suggested three and six months. At present however, there is conflicting evidence to support more prolonged or repeated counselling visits $[10,22]$. The London study investigating the pathway reported that only $30 \%$ of eligible patients returned for follow up which is similar to the generally high attrition rates seen in exercise schemes [12,23]. In Scotland, the need to improve formalised systems for recall and follow up of patients was identified [14] which are important considerations for successful implementation of the pathway.

\section{Additional training needs and resources}

Physiotherapists in this study endorsed the clinical feasibility of the pathway but only with the support of additional staffing, patient materials and training, all of which have significant cost implications. The estimated cost per patient completing the pathway in the UK is reported as $£ 53$ (GBP), for those recruited opportunistically, increasing to $£ 191$ at disease register sites, with the largest costs associated with training and initial set up [24]. Specific training needs were identified by participants particularly in the area of motivational interviewing and population approaches to screening. This supports recent findings which have highlighted deficits in physiotherapists' knowledge of PA, particularly around public health guidelines, patient education strategies and skills in behaviour change such as motivational interviewing $[5,25,26]$. Practitioners in England receive a two day training course in motivational interviewing to support delivery of the pathway and in Scotland the pathway is supported by an e-learning module and a 3.5 hour training session $[6,14]$. Results from the UK studies have concluded that even more in-depth and ongoing training may be required to support the programme $[12,13]$ as well as highlighting the importance of tailoring the training to reflect local services, the mix of practitioners and their prior knowledge of PA [14].

\section{Strengths and limitations}

There was an excellent response rate to this Delphi process, with the loss of only one participant in the final round. There was good representation from different professional grades adding to the heterogeneity of the sample and thus the validity of the Delphi process. Participants were highly experienced; over $70 \%$ were qualified for greater than ten years and many had significant clinical experience within primary care.

A number of limitations are acknowledged. A modification to the Delphi process was made whereby participants attended an education session at the start of the process. This was deemed necessary to allow for familiarisation with the pathway and to explain the study protocol. It cannot be assumed however, that there was not some discussion among participants and that opinions may have been altered in response. In addition, this was a regional sample, the majority of whom were female and employed in the public health service. There are limitations therefore, in extrapolating the results to the national physiotherapy population.

\section{Conclusion}

The Physical Activity Pathway "Let's Get Moving" was accepted as a clinically feasible option by primary care physiotherapists with some modifications and with the support of additional resources including staffing and education. At a time when there is ongoing commitment to develop primary care in Ireland, together with specific emphasis on disease prevention and health promotion, there is a unique opportunity to incorporate effective strategies for health promotion into health service provision. The positive response of physiotherapists supports the need for ongoing research to determine the acceptance of the pathway to a broader spectrum of primary care professionals as well as from the perspective of the service user.

\section{Acknowledgements}

The Physical Activity Clinical Pathway "Let's Get Moving" was developed by the Department of Health in England in collaboration with National Health Service London (NHS) and Natural England.

We would like to thank all of the participating physiotherapists for their time and input.

Ethical approval: Research Ethics Group of the Faculty of Health Sciences Trinity College Dublin.

Conflict of interest: None.

\section{References}

[1] World Health Organisation. Global Status Report on Noncommunicable Diseases 2010 Geneva. Switzerland: WHO Press; 2011.

[2] Balanda K, Barron S, Fahy L. Making chronic conditions count: hypertension, coronary heart disease, stroke, diabetes. A systematic approach to estimating and forecasting population prevalence on the island of Ireland. Executive Summary. Dublin: Institute of Public Health in Ireland; 2010.

[3] Department of Health. Ipsos MRBI. Healthy Ireland Survey 2015. Dublin; 2015.

[4] Department of Health. Healthy Ireland - A Framework for Improved Health and Wellbeing 2013-2025. Dublin: Department of Health; 2013.

[5] Barrett EM, Darker CD, Hussey J. Promotion of physical activity in primary care: knowledge and practice of general practitioners and physiotherapists. J Public Health 2012;21:63-9.

[6] Department of Health. Let's get moving - introducing a new physical activity care pathway - UK; 2010 http://webarchive.nationalarchives. gov.uk/+/www.dh.gov.uk/en/Publichealth/Healthimprovement/ PhysicalActivity/DH_099438 [accessed 06.09.13].

[7] National Institute for Health and Clinical Excellence. NICE Public Health Intervention Guidance no. 2. Four commonly used methods to increase physical activity; brief interventions in primary care, pedometers, exercise-referral schemes and community based exercise 
programmes for walking and cycling. London: National Institute for Health and Clinical Excellence; 2006.

[8] National Institute for Health and Care Excellence. NICE Guidance Physical activity: brief advice for adults in primary care. London: National Institute for Health and Care Excellence; 2013

[9] World Health Organisation. Global recommendations on physical activity for health. Geneva: World Health Organisation; 2010.

[10] Orrow G, Kinmonth A-L, Sanderson S, Sutton S. Effectiveness of physical activity promotion based in primary care: systematic review and meta-analysis of randomised controlled trials. BMJ 2012:344.

[11] Hardcastle SJ, Taylor AH, Bailey MP, Harley RA, Hagger MS. Effectiveness of a motivational interviewing intervention on weight loss, physical activity and cardiovascular disease risk factors: a randomised controlled trial with a 12-month post-intervention follow-up. Int J Behav Nutr Phys Act 2013;10:40.

[12] Bull FC, Milton KE. A process evaluation of a "physical activity pathway" in the primary care setting. BMC Public Health 2010;10:463.

[13] James D, Crone D, Curry N, Gidlow C. Report on the evaluation of the South Staffordshire Physical Activity Care Pathway pilot (short version). U.K: University of Gloucestershire; 2010.

[14] Jackson F, Myers F, Oates A, Martin N. NHS Primary Care Physical Activity Pathway. Feasibility Study. Final Main Report NHS Scotland; 2014.

[15] Hsu CC, Sandford BA. The Delphi technique: making sense of consensus. Pract Assess Res Eval 2007;12:1-8.

[16] Boulkedid R, Abdoul H, Loustau M, Sibony O, Alberti C. Using and reporting the Delphi method for selecting healthcare quality indicators: a systematic review. PLoS ONE 2011;6:e20476.

[17] McHugh ML. Interrater reliability: the kappa statistic. Biochem Med 2012;22:276-82

[18] Darker CD, Martin C, O’Dowd T, O’Kelly F, O’Kelly M, O’Shea B. A National Survey of Chronic Disease Management in Irish General
Practice. Dublin: Department of Public Health \& Primary Care Trinity College Dublin; 2011.

[19] Department of Health and Children. Tackling Chronic Disease. A Policy Framework for the Management of Chronic Diseases. Dublin: Department of Health; 2008.

[20] Heron N, Tully MA, McKinley MC, Cupples ME. Physical activity assessment in practice: a mixed methods study of GPPAQ use in primary care. BMC Fam Pract 2014;15:11.

[21] Bull F, Milton K, Boehler C. Report of the Evaluation of the Physical Activity Care Pathway London Feasibility Pilot - Final Technical Report. UK: BHFNC, SSES, Loughborough University; 2008.

[22] Lin JS, O'Connor E, Whitlock EP, Beil TL. Behavioral counseling to promote physical activity and a healthful diet to prevent cardiovascular disease in adults: a systematic review for the U.S. Preventive Services Task Force. Ann Intern Med 2010;153:736-50.

[23] Pavey T, Taylor A, Hillsdon M, Fox K, Campbell J, Foster C, et al. Levels and predictors of exercise referral scheme uptake and adherence: a systematic review. J Epidemiol Community Health 2012;66: 737-44.

[24] Boehler CE, Milton KE, Bull FC, Fox-Rushby JA. The cost of changing physical activity behaviour: evidence from a "physical activity pathway" in the primary care setting. BMC Public Health 2011; $11: 370$.

[25] O'Donoghue G, Cusack T, Doody C. Contemporary undergraduate physiotherapy education in terms of physical activity and exercise prescription: practice tutors' knowledge, attitudes and beliefs. Physiotherapy 2012;98:167-73.

[26] Aweto HA, Oligbo CN, Fapojuwo OA, Olawale OA. Knowledge, attitude and practice of physiotherapists towards promotion of physically active lifestyles in patient management. BMC Health Serv Res $2013 ; 13: 21$

Available online at www.sciencedirect.com

\section{ScienceDirect}

